\title{
Parity nonconserving observables in thermal neutron capture on a proton
}

\author{
C. H. Hyun ${ }^{a, b *}$, S. J. Lee ${ }^{a}$, J. Haidenbauer ${ }^{c}$, S. W. Hong ${ }^{a}$ \\ (a) Department of Physics and Institute of Basic Science, \\ Sungkyunkwan University, Suwon 440-746, Korea \\ (b) School of Physics, Seoul Nat'l University, Seoul 151-742, Korea \\ (c) Forschugszentrum Jülich, Institut für Kernphysik, D-52425 Jülich, Germany
}

(Dated: November 26, 2004)

\begin{abstract}
We calculate parity nonconserving observables in the processes where a neutron is captured on a proton at the threshold energy radiating a photon. Various potential models such as Paris, Bonn and Argonne $v 18$ are used for the strong interactions, and the meson-exchange description is employed for the weak interactions between hadrons. The photon polarization $P_{\gamma}$ in the unpolarized neutron capture process and photon asymmetry $A_{\gamma}$ in the polarized neutron capture process are obtained in terms of the weak meson-nucleon coupling constants. $A_{\gamma}$ turns out to be basically insensitive to the employed strong interaction models and thus can be uniquely determined in terms of the weak coupling constants, but $P_{\gamma}$ depends significantly on the strong interaction models.
\end{abstract}

\footnotetext{
* e-mail: hch@meson.skku.ac.kr
} 


\section{INTRODUCTION}

Recent experiments to explore the weak interactions between hadrons through parity nonconserving (PNC) observables in nuclear systems [1, 2] or reactions [3, 4] have triggered revived interests in this field. These PNC observables can be related to the meson-nucleon weak coupling constants which are introduced in the meson-exchange potential description of the hadronic weak interaction [5]. However, due to various uncertainties (see Ref. [5] for details), the weak coupling constants were fixed only within certain ranges [5]. Thus, it has been hoped that the PNC observables from various experiments can reduce the range of these coupling constants and eventually determine the values. The situation, however, has not been much improved even by the recent measurements. For example, the value of the $\pi-N$ weak coupling constant, $h_{\pi}^{1}$, from the anapole moment of ${ }^{133} \mathrm{Cs}$ [1] is inconsistent with a previous value obtained from the forbidden $\gamma$-decay of ${ }^{18} \mathrm{~F}[6]: h_{\pi}^{1}$ determined by the former is larger than that from the latter by a factor of 7 . New experiments, already completed [3], being done [4] or expected to be performed, concern two-nucleon systems in which many body effects are absent. Thus they are expected to give more stringent constraints on the weak coupling constants. For the current status of research on the weak coupling constants, see 7].

In this work, we calculate the photon asymmetry $A_{\gamma}$ in the radiative capture of a polarized neutron on a proton, $\vec{n}+p \rightarrow d+\gamma$, and the circular polarization of photons $P_{\gamma}$ in $n+p \rightarrow d+\gamma$ at the threshold. The latest experimental value of $A_{\gamma}$ is $-(1.5 \pm 4.8) \times 10^{-8}$ [8], but the experiment being done at LANSCE aims at the accuracy of $10^{-9}[4]$. Theoretical calculations of $A_{\gamma}$ using strong models made in the 1960's and the 1970's such as Hamada-Johnston, Reidsoft-core and Tourreil-Sprung show results similar to each other; $A_{\gamma} \simeq-0.11 h_{\pi}^{1}[9]$. $A_{\gamma}$ is predominantly determined by $h_{\pi}^{1}$ and depends very little on other coupling constants (as will be shown in Table (I). In this work we present $A_{\gamma}$ calculated with potentials such as Paris [10], Bonn [11], Bonn-A and Bonn-B [12], and Argonne v18 (Av18) 13]. We compare our results with previous ones [9] and investigate the model dependence of $A_{\gamma}$.

Contrary to $A_{\gamma}, P_{\gamma}$ at the threshold is known to be sensitive to the heavy meson ( $\rho$ and $\omega)$ components of the weak potentials [9, 14]. The most recent experimental value of $P_{\gamma}$ is $(1.8 \pm 1.8) \times 10^{-7}[15]$, and theoretical calculations made in the 1970's agree with this value within the experimental errors. However, since $P_{\gamma}$ is sensitive to the short range properties 
of the strong interactions as well as of the weak interactions, its model dependence is more noticeable than $A_{\gamma}[14,16]$. Since the inverse process, $\vec{\gamma}+d \rightarrow n+p$, whose PNC asymmetry at the threshold is equal to $P_{\gamma}$, becomes experimentally feasible nowadays, we expect that $P_{\gamma}$ can be measured more precisely and can provide more constraints on the weak dynamics of hadrons. We thus investigate the model dependence of $P_{\gamma}$ with the same potentials that we use in calculating $A_{\gamma}$.

In Sect. 2, we present the Desplanques-Donaghue-Holstein (DDH) potential [5] and the parity-admixed wave functions in the initial and the final states. In Sect. 3, the electromagnetic operators are presented, matrix elements are derived, and the results for $A_{\gamma}$ and $P_{\gamma}$ are shown. Discussions on the results follow in Sect. 4.

\section{PARITY ADMIXED WAVE FUNCTION}

The Schrödinger equation for a two-nucleon system can be written as

$$
\begin{aligned}
H \Psi(\boldsymbol{r}) & =\left[-\frac{1}{m_{N}}\left(\frac{1}{r} \frac{\partial^{2}}{\partial r^{2}} r-\frac{l(l+1)}{r^{2}}\right)+V_{C}(r)+V_{T}(r) S_{12}(\hat{\boldsymbol{r}})+V_{p n c}(\boldsymbol{r})\right] \Psi(\boldsymbol{r}) \\
& =E \Psi(\boldsymbol{r})
\end{aligned}
$$

where $V_{T}$ represents the tensor potential and $V_{C}$ includes central, spin-orbit, spin-spin and quadratic spin-orbit interactions in the strong potential. In the Paris and Bonn potentials, it is essential to include the momentum dependent term in the central potential to obtain the correct phase shifts even at low energies. In Ref. [17] a transformation useful for treating the momentum dependent term is suggested. In this work, however, we have dealt with the momentum dependent terms without using such a transformation and have solved the Schrödinger equation as it is. We have confirmed that the solutions thus obtained reproduce the results of each potential model [10, 11, 12] fairly well with differences less than 1\%. The small differences can be attributed to the use of slightly different values of physical quantities in the calculations. $V_{p n c}$ is the PNC potential, and we use the one given by DDH [5]

$$
\begin{aligned}
V_{p n c}(\boldsymbol{r}) & =V_{p n c}^{\pi}(\boldsymbol{r})+V_{p n c}^{\rho}(\boldsymbol{r})+V_{p n c}^{\omega}(\boldsymbol{r}), \\
V_{p n c}^{\pi}(\boldsymbol{r}) & =i \frac{g_{\pi N N} h_{\pi}^{1}}{2 \sqrt{2} m_{N}}\left(\boldsymbol{\tau}_{1} \times \boldsymbol{\tau}_{2}\right)^{z}\left(\boldsymbol{\sigma}_{1}+\boldsymbol{\sigma}_{2}\right) \cdot\left[\boldsymbol{p}, f_{\pi}(r)\right] \\
V_{p n c}^{\rho}(\boldsymbol{r}) & =-\frac{g_{\rho N N}}{m_{N}}\left[\left(h_{\rho}^{0} \boldsymbol{\tau}_{1} \cdot \boldsymbol{\tau}_{2}+\frac{1}{2} h_{\rho}^{1}\left(\tau_{1}^{z}+\tau_{2}^{z}\right)+\frac{h_{\rho}^{2}}{2 \sqrt{6}}\left(3 \tau_{1}^{z} \tau_{2}^{z}-\boldsymbol{\tau}_{1} \cdot \boldsymbol{\tau}_{2}\right)\right) \times\right.
\end{aligned}
$$




$$
\begin{aligned}
& \left(\left(\boldsymbol{\sigma}_{1}-\boldsymbol{\sigma}_{2}\right) \cdot\left\{\boldsymbol{p}, f_{\rho}(r)\right\}+i\left(1+\chi_{\rho}\right)\left(\boldsymbol{\sigma}_{1} \times \boldsymbol{\sigma}_{2}\right) \cdot\left[\boldsymbol{p}, f_{\rho}(r)\right]\right) \\
+ & \left.i \frac{h_{\rho}^{1^{\prime}}}{2}\left(\boldsymbol{\tau}_{1} \times \boldsymbol{\tau}_{2}\right)^{z}\left(\boldsymbol{\sigma}_{1}+\boldsymbol{\sigma}_{2}\right) \cdot\left[\boldsymbol{p}, f_{\rho}(r)\right]-\frac{h_{\rho}^{1}}{2}\left(\tau_{1}^{z}-\tau_{2}^{z}\right)\left(\boldsymbol{\sigma}_{1}+\boldsymbol{\sigma}_{2}\right) \cdot\left\{\boldsymbol{p}, f_{\rho}(r)\right\}\right], \\
V_{p n c}^{\omega}(\boldsymbol{r})=- & \frac{g_{\omega N N}}{m_{N}}\left[\left(h_{\omega}^{0}+\frac{1}{2} h_{\omega}^{1}\left(\tau_{1}^{z}+\tau_{2}^{z}\right)\right) \times\right. \\
& \left(\left(\boldsymbol{\sigma}_{1}-\boldsymbol{\sigma}_{2}\right) \cdot\left\{\boldsymbol{p}, f_{\omega}(r)\right\}+i\left(1+\chi_{\omega}\right)\left(\boldsymbol{\sigma}_{1} \times \boldsymbol{\sigma}_{2}\right) \cdot\left[\boldsymbol{p}, f_{\omega}(r)\right]\right) \\
+ & \left.\frac{h_{\omega}^{1}}{2}\left(\tau_{1}^{z}-\tau_{2}^{z}\right)\left(\boldsymbol{\sigma}_{1}+\boldsymbol{\sigma}_{2}\right) \cdot\left\{\boldsymbol{p}, f_{\omega}(r)\right\}\right]
\end{aligned}
$$

where the strong coupling constants are $g_{\pi N N}=13.45, g_{\rho N N}=2.79, g_{\omega N N}=8.37$ and the anomalous magnetic moments are $\chi_{\rho}=3.71$ and $\chi_{\omega}=-0.12$. The Yukawa functions $f_{M}(r)$ are defined as

$$
f_{M}(r)=\frac{\mathrm{e}^{-m_{M} r}}{4 \pi r}, \quad(M=\pi, \rho, \omega)
$$

The quantities $h_{M}^{\Delta I}$ represent the weak meson-nucleon coupling constants where $\Delta I$ denotes the isospin transfer.

At the threshold energy, the initial scattering state, $n+p$, is dominated by the lowest angular momentum state, i.e., the ${ }^{1} S_{0}$ channel, and higher angular momentum states are suppressed. Thus in this work we just include the next low-lying state, the ${ }^{3} S_{1}-{ }^{3} D_{1}$ partial waves, where the ${ }^{3} D_{1}$ state is induced by the tensor interaction in the initial scattering state. Then the parity-even state of the initial wave function consists of the ${ }^{1} S_{0},{ }^{3} S_{1}$ and ${ }^{3} D_{1}$ states.

Since $V_{p n c}$ is a parity-odd operator, it creates opposite parity components in the wave function. For example, when $V_{p n c}$ is operated on the ${ }^{1} S_{0}$ state, the isoscalar and isotensor terms of $V_{p n c}$ generate a ${ }^{3} \tilde{P}_{0}$ admixture, where the tilde denotes the parity-admixed components generated from the DDH potential. Similarly, ${ }^{3} \tilde{P}_{1}$ and ${ }^{1} \tilde{P}_{1}$ admixtures arise from applying the isovector and isoscalar components of $V_{p n c}$ to the ${ }^{3} S_{1}-{ }^{3} D_{1}$ state, respectively. The total wave function of the initial state with its parity admixture at the threshold can be written as

$$
\begin{aligned}
\Psi_{i}(\boldsymbol{r}) & =\Psi_{i}^{p c}(\boldsymbol{r})+\Psi_{i}^{p n c}(\boldsymbol{r}) \\
\Psi_{i}^{p c}(\boldsymbol{r}) & =\frac{1}{\sqrt{4 \pi} r}\left[u_{s}(r) \chi_{00} \zeta_{10}+\left(u_{t}(r)+\frac{S_{12}(\hat{\boldsymbol{r}})}{\sqrt{8}} w_{t}(r)\right) \chi_{1 S_{z}} \zeta_{00}\right] \\
\Psi_{i}^{p n c}(\boldsymbol{r}) & =-\frac{i}{\sqrt{4 \pi} r}\left[\sqrt{\frac{3}{8}} \tilde{v}_{t}^{3 p 1}(r)\left(\boldsymbol{\sigma}_{1}+\boldsymbol{\sigma}_{2}\right) \chi_{1 S_{z}} \zeta_{10}+\frac{1}{2} \tilde{v}_{t}^{3 p 0}(r)\left(\boldsymbol{\sigma}_{1}-\boldsymbol{\sigma}_{2}\right) \chi_{00} \zeta_{10}\right] \cdot \hat{\boldsymbol{r}},
\end{aligned}
$$


where $\chi_{S S_{z}}$ and $\zeta_{T T_{z}}$ represent the spin and isospin part, respectively. $u_{s}$ is the radial part of the wave function for the ${ }^{1} S_{0}$ channel, $u_{t}$ for ${ }^{3} S_{1}$ and $w_{t}$ for ${ }^{3} D_{1}$. The final state wave function can be written in a similar way as

$$
\begin{aligned}
\Psi_{f}(\boldsymbol{r}) & =\Psi_{f}^{p c}(\boldsymbol{r})+\Psi_{f}^{p n c}(\boldsymbol{r}), \\
\Psi_{f}^{p c}(\boldsymbol{r}) & =\frac{1}{\sqrt{4 \pi} r}\left(u_{d}(r)+\frac{S_{12}(\hat{\boldsymbol{r}})}{\sqrt{8}} w_{d}(r)\right) \chi_{1 S_{z}} \zeta_{00}, \\
\Psi_{f}^{p n c}(\boldsymbol{r}) & =\frac{i}{\sqrt{4 \pi} r}\left[\frac{\sqrt{3}}{2} \tilde{v}_{d}^{1 p 1}(r)\left(\boldsymbol{\sigma}_{1}-\boldsymbol{\sigma}_{2}\right) \chi_{1 S_{z}} \zeta_{00}-\sqrt{\frac{3}{8}} \tilde{v}_{d}^{3 p 1}(r)\left(\boldsymbol{\sigma}_{1}+\boldsymbol{\sigma}_{2}\right) \chi_{1 S_{z}} \zeta_{10}\right] \cdot \hat{\boldsymbol{r}},
\end{aligned}
$$

where $u_{d}(r)\left(w_{d}(r)\right)$ is the radial wave function for the ${ }^{3} S_{1}\left({ }^{3} D_{1}\right)$ deuteron state, and $\tilde{v}_{d}^{1 p 1}$ and $\tilde{v}_{d}^{3 p 1}$ denote the parity nonconserving admixture due to the ${ }^{1} \tilde{P}_{1}$ and ${ }^{3} \tilde{P}_{1}$ states, respectively. By inserting the initial and the final wave functions into the Schrödinger equation (11) with the strong and weak PNC potentials, one can obtain the radial wave equation for each channel (see Appendix for details).

\section{MATRIX ELEMENTS, $P_{\gamma}$ AND $A_{\gamma}$}

At the threshold energy, it is well known that the neutron capture cross section is dominated by the isovector M1 transition. We can evaluate the parity conserving M1 transition amplitude by using the one-body spin current operator

$$
\boldsymbol{J}_{M}=-i \frac{\mu_{V}}{4 m_{N}} \sum_{i} \tau_{i}^{z} \boldsymbol{\sigma}_{i} \times \boldsymbol{k}_{\gamma}
$$

where $\mu_{V}=4.71$, and $\boldsymbol{k}_{\gamma}$ is the photon momentum. Amplitudes between the states with opposite parities would become non-zero through the E1 transition. While the impulse approximation is used in evaluating the M1 amplitude, the contribution from the exchange currents can be well accounted for by the Siegert's theorem. The E1 current operator with Siegert's theorem reads

$$
\boldsymbol{J}_{E}^{S}=-i \frac{\omega}{4}\left(\tau_{1}^{z}-\tau_{2}^{z}\right) \boldsymbol{r},
$$

where $\omega$ is the photon energy $\left(2.2246 \mathrm{MeV}\right.$ at threshold). The transition amplitudes $\left(\mathcal{M}_{i}^{f}\right)$ can be classified in terms of the electromagnetic type $\mathcal{M}(=M$ or $E)$, the initial $(i)$ and the final $(f)$ states. The leading parity-conserving isovector M1 transition occurs between the initial ${ }^{1} S_{0}$ and final ${ }^{3} S_{1}$ states, and we denote its amplitude by $M_{1 s 0}^{3 s 1}$. The non-zero PNC

E1 amplitudes are represented similarly as $\tilde{E}_{3 p 0}^{3 s 1}$ for ${ }^{3} \tilde{P}_{0} \rightarrow{ }^{3} S_{1}+{ }^{3} D_{1}, \tilde{E}_{1 s 0}^{1 p 1}$ for ${ }^{1} S_{0} \rightarrow{ }^{1} \tilde{P}_{1}$, 
$\tilde{E}_{3 p 1}^{3 s 1}$ for ${ }^{3} \tilde{P}_{1} \rightarrow{ }^{3} S_{1}+{ }^{3} D_{1}$, and $\tilde{E}_{3 s 1}^{3 p 1}$ for ${ }^{3} S_{1}+{ }^{3} D_{1} \rightarrow{ }^{3} \tilde{P}_{1}$, where the tildes are to distinguish the PNC amplitudes from the normal parity conserving ones. With the wave functions of Eqs. (5) - (8) , we obtain the matrix elements

$$
\begin{aligned}
M_{1 s 0}^{3 s 1} & =\frac{\omega \mu_{V}}{4 m_{N}} \int d r u_{d}(r) u_{s}(r), \\
\tilde{E}_{3 p 0}^{3 s 1} & =\frac{\omega}{12} \int d r r\left(u_{d}(r)-\sqrt{2} w_{d}(r)\right) \tilde{v}_{t}^{3 p 0}(r), \\
\tilde{E}_{1 s 0}^{1 p 1} & =\frac{\omega}{4 \sqrt{3}} \int d r r \tilde{v}_{d}^{1 p 1}(r) u_{s}(r), \\
\tilde{E}_{3 p 1}^{3 s 1} & =-\frac{\omega}{4 \sqrt{6}} \int d r r\left(u_{d}(r)+\frac{w_{d}(r)}{\sqrt{2})} \tilde{v}_{t}^{3 p 1},\right. \\
\tilde{E}_{3 s 1}^{3 p 1} & =\frac{\omega}{4 \sqrt{6}} \int d r r \tilde{v}_{d}^{3 p 1}\left(u_{t}(r)+\frac{w_{t}(r)}{\sqrt{2}}\right) .
\end{aligned}
$$

In terms of these electromagnetic amplitudes, the two PNC observables are written as

$$
\begin{aligned}
& A_{\gamma}=-2 \frac{\tilde{E}_{3 p 1}^{3 s 1}+\tilde{E}_{3 s 1}^{3 p 1}}{M_{1 s 0}^{3 s 1}} \equiv A_{\gamma}^{i}+A_{\gamma}^{f}, \\
& P_{\gamma}=-2 \frac{\tilde{E}_{3 p 0}^{3 s 1}+\tilde{E}_{1 s 0}^{1 p 1}}{M_{1 s 0}^{3 s 1}} \equiv P_{\gamma}^{i}+P_{\gamma}^{f},
\end{aligned}
$$

where $A_{\gamma}^{i} \equiv-2 \tilde{E}_{3 p 1}^{3 s 1} / M_{1 s 0}^{3 s 1}$ and $P_{\gamma}^{i} \equiv-2 \tilde{E}_{3 p 0}^{3 s 1} / M_{1 s 0}^{3 s 1}$ have the PNC component in the initial state and $A_{\gamma}^{f} \equiv-2 \tilde{E}_{3 s 1}^{3 p 1} / M_{1 s 0}^{3 s 1}$ and $P_{\gamma}^{f} \equiv-2 \tilde{E}_{1 s 0}^{1 p 1} / M_{1 s 0}^{3 s 1}$ have the PNC component in the final state. Numerical results are given in Table \. We express the results for $A_{\gamma}$ and $P_{\gamma}$ in terms

of the weak coupling constants $h_{M}^{\Delta I}$ to show explicitly the dependence of $A_{\gamma}$ and $P_{\gamma}$ on each meson. "Best values" refer to $A_{\gamma}$ and $P_{\gamma}$ values evaluated with the so-called best values of the weak meson-coupling constants suggested by Ref. [5]. They are $h_{\rho}^{0}=-11.4, h_{\omega}^{0}=-1.9$ $h_{\rho}^{2}=-9.5, h_{\pi}^{1}=4.6, h_{\rho}^{1}=-0.2$ and $h_{\omega}^{1}=-1.1$, in units of $10^{-7}$.

\section{RESULTS AND DISCUSSIONS}

\section{Asymmetry $\left(\boldsymbol{A}_{\gamma}\right)$}

As shown in Table I the Bonn and Av18 models predict the same $A_{\gamma}$ value, while the best value from the Paris potential is larger in magnitude than those from Bonn and Av18 by a factor of 1.27 . This factor can be understood by examining the wave functions that contribute to $A_{\gamma}$. $u_{s}$ and $u_{d}$ are plotted in Fig. 1, and $\tilde{v}_{d}^{3 p 1}$ in Fig. 2, As can be seen in Fig. 1] and 2. $u_{d}$ and $\tilde{v}_{d}^{3 p 1}$ calculated with different potentials are very similar to each other for all potentials, but $u_{s}$ calculated with the Paris potential is substantially different from $u_{s}$ from 


\begin{tabular}{|c|c|c|c|c|}
\hline & & \multicolumn{3}{|c|}{ Best values $\left(\times 10^{-8}\right)$} \\
\cline { 3 - 5 } Model & $A_{\gamma}$ & $A_{\gamma}$ & $A_{\gamma}^{i}$ & $A_{\gamma}^{f}$ \\
\hline Paris & $-0.148 h_{\pi}^{1}-0.001 h_{\rho}^{1}+0.003 h_{\omega}^{1}$ & -6.85 & -3.34 & -3.51 \\
\hline Bonn & $-0.117 h_{\pi}^{1}-0.001 h_{\rho}^{1}+0.003 h_{\omega}^{1}$ & -5.42 & -2.66 & -2.76 \\
\hline Bonn-B & $-0.117 h_{\pi}^{1}-0.001 h_{\rho}^{1}+0.002 h_{\omega}^{1}$ & -5.41 & -2.65 & -2.76 \\
\hline Av18 & $-0.117 h_{\pi}^{1}-0.001 h_{\rho}^{1}+0.002 h_{\omega}^{1}$ & -5.41 & -2.63 & -2.78 \\
\hline \hline & & Best values $\left(\times 10^{-8}\right)$ \\
\cline { 3 - 5 } Model & $P_{\gamma}$ & $P_{\gamma}$ & $P_{\gamma}^{i}$ & $P_{\gamma}^{f}$ \\
\hline Paris & $-0.0106 h_{\rho}^{0}+0.0074 h_{\omega}^{0}-0.0191 h_{\rho}^{2}$ & 2.88 & -1.24 & 4.12 \\
\hline Bonn & $-0.0890 h_{\rho}^{0}+0.0088 h_{\omega}^{0}-0.0214 h_{\rho}^{2}$ & 12.01 & -1.40 & 13.4 \\
\hline Bonn-B & $-0.0286 h_{\rho}^{0}+0.0012 h_{\omega}^{0}-0.0208 h_{\rho}^{2}$ & 5.21 & -1.35 & 6.56 \\
\hline A $v 18$ & $-0.0088 h_{\rho}^{0}+0.0034 h_{\omega}^{0}-0.0175 h_{\rho}^{2}$ & 2.64 & -1.11 & 3.75 \\
\hline
\end{tabular}

TABLE I: Results for the observables $A_{\gamma}$ and $P_{\gamma}$ for various phenomenological models in terms of the weak coupling constants $h_{M}^{\Delta I}$. Best values mean $A_{\gamma}$ and $P_{\gamma}$ values obtained with the best values of $h_{M}^{\Delta I}$ suggested by DDH. They are in units of $10^{-8}$. The definitions of $P_{\gamma}^{i, f}$ and $A_{\gamma}^{i, f}$ are given in Eqs. (17) and (16), respectively.
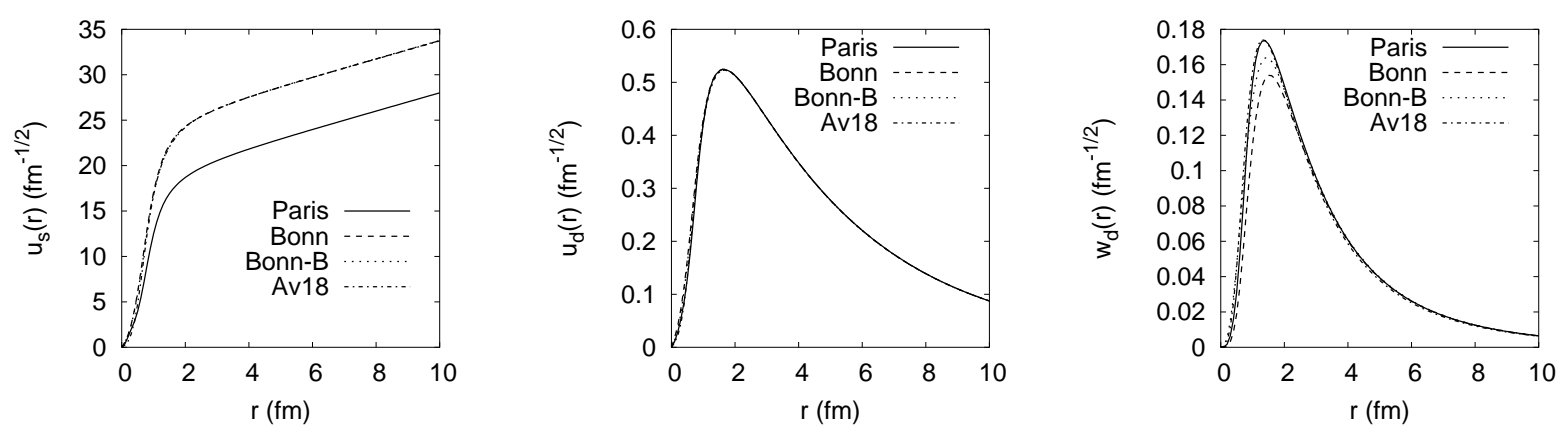

FIG. 1: The wave functions $u_{s}(r), u_{d}(r)$ and $w_{d}(r)$ calculated with different potentials are plotted. The results for $u_{s}(r)$ from Bonn, Bonn-B and Av18 are indistinguishable and correspond to the upper curve in the figure. $u_{t}(r)$ and $w_{t}(r)$ are not shown here, because they do not depend very much on the models. 

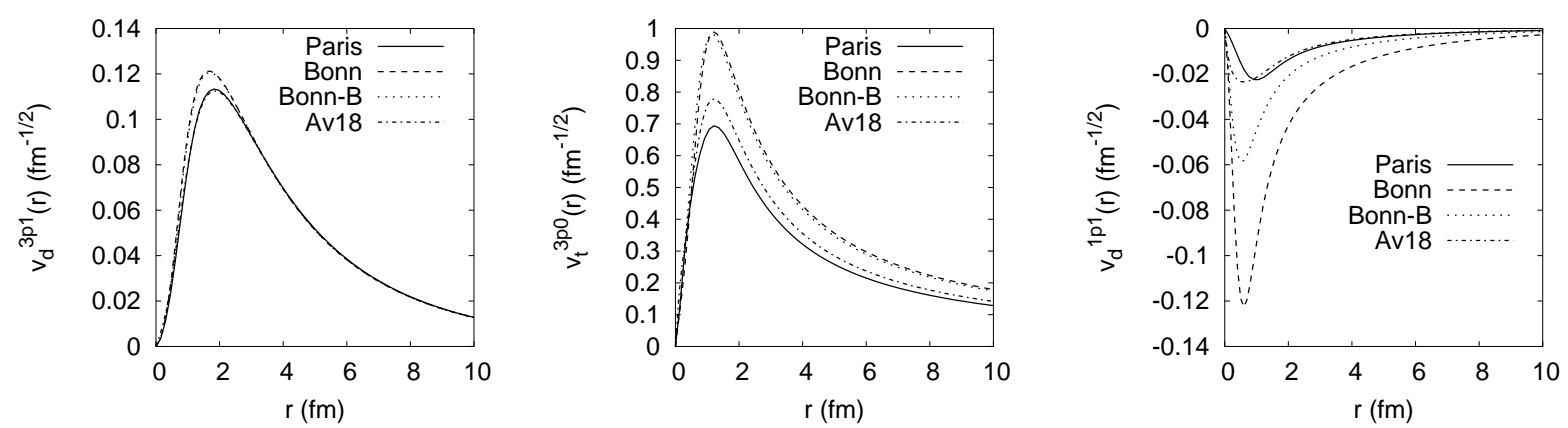

FIG. 2: Wave functions for the parity-admixed states for different potentials. The wave functions are given in units of $h_{\pi}^{1}$. Note the difference in the scale.

other potentials. $\left(\tilde{v}_{t}^{3 p 1}\right.$, though not shown here, is more or less the same for all potential models.) The reason for this difference can be traced back to the fact that the Paris potential was fitted to proton-proton data and therefore yields a scattering length of $a=-17.6 \mathrm{fm}$ for the ${ }^{1} S_{0}$ channel (in the absence of the Coulomb interaction) while A $v 18$ and Bonn are neutron-proton models and yield $a=-23.7 \mathrm{fm}$. The M1 isovector amplitude $M_{1 s 0}^{3 s 1}$ in the denominator of Eqs. (16) and (17) is 0.184 for Paris potential and 0.233 for Av18 and Bonn potentials. Since the wave functions that contribute to the E1 amplitude in Eqs. (14) and (15) (numerator of $A_{\gamma}$ ) are very similar, the difference in the values of $A_{\gamma}$ comes mostly from the value of the M1 amplitude in the denominator of $A_{\gamma}$. Indeed, the ratio of the M1 amplitudes for Av18 to Paris, 1.27, is equal to the ratio of the best value of $A_{\gamma}$. Thus, if one could readjust the Paris potential to produce the accepted $n-p$ scattering length in the ${ }^{1} S_{0}$ channel, the four models would give us essentially model-independent $A_{\gamma}$ values. A recent work [18] in which various contributions from the exchange currents are taken into account confirms this model-independent nature of $A_{\gamma}$. The magnitude of $A_{\gamma}$ in Ref. [18] $\left(A_{\gamma}=-4.98 \times 10^{-8}\right)$ with pion-exchange currents is smaller than ours $\left(A_{\gamma}=-5.41 \times 10^{-8}\right)$ by about $9 \%$, and is in agreement with the result of Ref. [19] $\left(A_{\gamma}=-4.94 \times 10^{-8}\right)$ where one-body and leading pion-exchange currents are considered. On the other hand, if one employs the $h_{\pi}^{1}$ value from the ${ }^{18} \mathrm{~F}$ [6] and ${ }^{133} \mathrm{Cs}$ [1] experiments, $A_{\gamma}$ becomes $-1.52 \times 10^{-8}$ and $-11.1 \times 10^{-8}$, respectively. Since the contribution from the pion to $A_{\gamma}$ is more than $99 \%$ of the total value (see Table 【), a more accurate measurement of $A_{\gamma}$ can provide a stringent determination of $h_{\pi}^{1}$. 

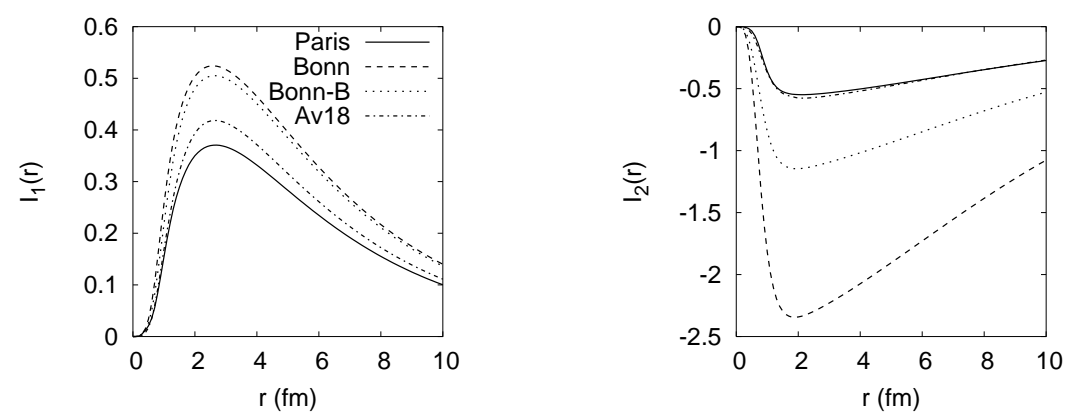

FIG. 3: Integrands that enter into the E1 amplitude of $P_{\gamma}$. The left panel shows $I_{1}(r) \equiv r\left(u_{d}(r)-\right.$ $\left.\sqrt{2} w_{d}(r)\right) \tilde{v}_{t}^{3 p 0}(r)$ of Eq. (12), and the right one $I_{2}(r) \equiv r \tilde{v}_{d}^{1 p 1}(r) u_{s}(r)$ of Eq. (13).

In passing, we remark that $A_{\gamma}$ from a previous work [20] using Paris potential differs from our $A_{\gamma}$ in sign though the magnitudes agree. It appears that the definitions of $A_{\gamma}$ differ in sign.

\section{Polarization $\left(\boldsymbol{P}_{\gamma}\right)$}

While $A_{\gamma}$ is dominated by the long range part of the interactions and is practically modelindependent, $P_{\gamma}$ depends strongly on the heavy meson exchanges and on the potential model. $P_{\gamma}$ 's calculated with the best values of the weak coupling constants [5] and Paris and Av18 potentials are similar to each other, but $P_{\gamma}$ 's evaluated with Bonn and Bonn-B are larger than that with A $v 18$ by a factor of 5 and 2, respectively. (Bonn-A and Bonn produce similar results and thus Bonn-A is not included in the discussion.) $P_{\gamma}$ 's expressed in terms of $h_{M}^{\Delta I}$ in Table【 show that $P_{\gamma}$ from Bonn is more sensitive to $h_{\rho}^{0}$ than $P_{\gamma}$ from other potentials, while the terms depending on $h_{\omega}^{0}$ and $h_{\rho}^{2}$ are only moderately model dependent. The contributions from the initial $\left({ }^{3} \tilde{P}_{0}\right)$ and the final $\left({ }^{1} \tilde{P}_{1}\right)$ states listed in Table show that the initial state contribution $\left(P_{\gamma}^{i}\right)$ is rather model-independent, but the contribution from the final state $\left(P_{\gamma}^{f}\right)$ is highly dependent on the potentials.

The numerical factors in front of the weak coupling constants in Table $\llbracket$ are determined by the strong potentials in each channel through the wave functions of the ${ }^{1} S_{0}$ and ${ }^{3} S_{1}-{ }^{3} D_{1}$ channels that enter into the source terms in the Schrödinger equation of the parity-admixed states (see Appendix). The wave functions for the ${ }^{3} \tilde{P}_{0}$ and the ${ }^{1} \tilde{P}_{1}$ states are shown in Fig. 2, and the corresponding integrands of the E1 amplitudes, Eqs. (12) and (13), are shown in Fig. 3. The wave function $\left(\tilde{v}_{t}^{3 p 0}(r)\right)$ and the integrand $\left(I_{1}(r)\right)$ for the ${ }^{3} \tilde{P}_{0}$ state exhibit a sizeable model dependence. However, for the ${ }^{1} \tilde{P}_{1}$ channel there are even more 


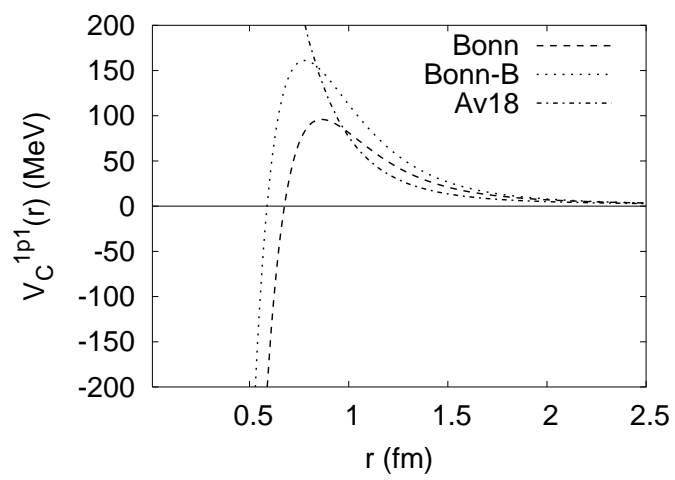

FIG. 4: Comparison of the central potentials in the ${ }^{1} P_{1}$ channel of the Bonn, Bonn-B, and Av18 potentials.
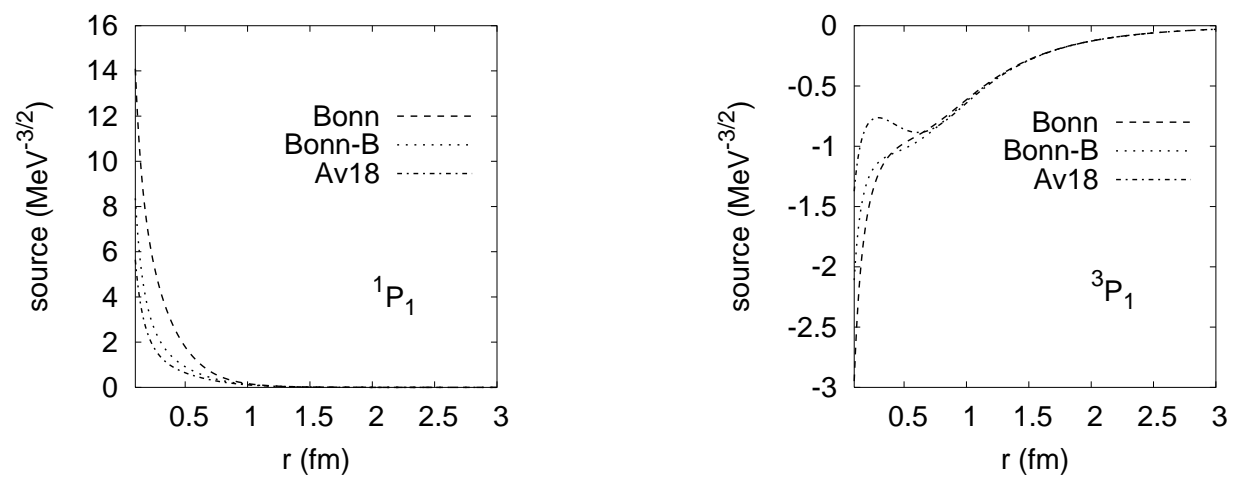

FIG. 5: Source terms for the ${ }^{1} \tilde{P}_{1}$ and ${ }^{3} \tilde{P}_{1}$ states. Compared to the terms of ${ }^{3} P_{1}$, those of ${ }^{1} P_{1}$ are more model dependent. Note the difference in the scale.

drastic variations in both wave function $\left(\tilde{v}_{d}^{1 p 1}(r)\right)$ and the integrand $\left(I_{2}(r)\right)$ depending on different potentials. Such a strong model dependency can be understood from the behavior of the strong potential in the ${ }^{1} P_{1}$ channel and the source term that contributes to $\tilde{v}_{d}^{1 p 1}$ in the Schrödinger equation (see Eq. (A.6) ). Fig. 目 shows the strong potentials in the ${ }^{1} P_{1}$ channel. The Bonn potential for ${ }^{1} P_{1}$ channel becomes attractive in the short range region while A $v 18$ is repulsive in the whole region. The attraction at short ranges increases the probability for a nucleon to be present in the region, and this can partly explain the shape of $\tilde{v}_{d}^{1 p 1}$ in Fig. 2. A recent work by $\mathrm{R}$. Schiavilla et al. shows a similar behavior of $\tilde{v}_{d}^{1 p 1}[18]$.

In Fig. [5, we compare the source terms of the ${ }^{3} \tilde{P}_{1}$ (the right hand side of Eq. (A.5) ) and ${ }^{1} \tilde{P}_{1}$ (the right hand side of Eq. (A.6) ) states. The sources for the ${ }^{3} \tilde{P}_{1}$ state from different models 
show a moderate model dependence for $r \leq 0.5 \mathrm{fm}$, but they have significant magnitudes and are indistinguishable in the intermediate and long range regions, which explains the model independent results of $A_{\gamma}$ in Table【 On the contrary, most of the contribution to the source terms of $\tilde{v}_{d}^{1 p 1}$ comes from the intermediate and short range region, and they depend strongly on the model. A larger source combined with attraction in the short-range region, as it is the case for the ${ }^{1} \tilde{P}_{1}$ channel of the Bonn models, yields an enhanced contribution to $P_{\gamma}$.

Concluding, we have calculated parity non-conserving observables $P_{\gamma}$ for the reaction $n+p \rightarrow d+\gamma$ and $A_{\gamma}$ for the reaction $\vec{n}+p \rightarrow d+\gamma$ at threshold. We have employed the Paris, Bonn and Av18 potentials for the strong interaction and the DDH potential for the weak interaction. $A_{\gamma}$ turns out to be independent of the strong interaction models, while $P_{\gamma}$ is sensitive to the dynamics at short ranges. Since $A_{\gamma}$ is rather strong-interaction independent, one can reduce the uncertainty in the value of $h_{\pi}^{1}$ by measuring $A_{\gamma}$ accurately. Regarding $P_{\gamma}$, there are relatively large uncertainties, which stem from ambiguities in both strong and weak interactions at short ranges. However, since the major uncertainty comes from the ${ }^{1} \tilde{P}_{1}$ channel and the value of $h_{\rho}^{0}$, an accurate experimental measurement of $P_{\gamma}$ can shed some light on the weak coupling constants.

\section{Acknowledgments}

We thank B. Desplanques for useful discussions. The work is supported by the Korea Research Foundation Grant (KRF-2003-070-C00015).

\section{APPENDIX}

The radial equations for the ${ }^{1} S_{0}$ continuum, and the ${ }^{3} S_{1}-{ }^{3} D_{1}$ continuum and bound states read

$$
\begin{aligned}
u_{s}^{\prime \prime}(r)+m_{N}\left(E-V_{C}(r)\right) u_{s}(r) & =0 \\
u_{t(d)}^{\prime \prime}(r)+m_{N}\left(E-V_{C}(r)\right) u_{t(d)}(r) & =\sqrt{8} m_{N} V_{T}(r) w_{t(d)}(r), \\
w_{t(d)}^{\prime \prime}(r)-\frac{6}{r^{2}} w_{t(d)}(r)-m_{N}\left(E-V_{C}(r)+2 V_{T}(r)\right) w_{t(d)}(r) & =\sqrt{8} m_{N} V_{T}(r) u_{t(d)}(r) .
\end{aligned}
$$

The equations for the parity-admixed states are

$$
\tilde{v}_{t}^{3 p 0^{\prime \prime}}(r)-\frac{2}{r^{2}} \tilde{v}_{t}^{3 p 0}+m_{N}\left(E-V_{C}(r)+4 V_{T}(r)\right) \tilde{v}_{t}^{3 p 0}=
$$




$$
\begin{aligned}
& -2\left[\left(\chi_{\rho}+2\right) u_{s}(r) \frac{\partial}{\partial r}\left(F_{\rho}^{0}(r)-\sqrt{\frac{2}{3}} F_{\rho}^{2}(r)\right)+\left(\chi_{\omega}+2\right) u_{s}(r) \frac{\partial}{\partial r} F_{\omega}^{0}(r)\right. \\
& \left.+2 r\left(F_{\rho}^{0}(r)-\sqrt{\frac{2}{3}} F_{\rho}^{2}(r)+F_{\omega}^{0}(r)\right) \frac{\partial}{\partial r}\left(\frac{u_{s}(r)}{r}\right)\right], \\
& \tilde{v}_{t(d)}^{3 p 1^{\prime \prime}}(r)-\frac{2}{r^{2}} \tilde{v}_{t(d)}^{3 p 1}(r)+m_{N}\left(E-V_{C}(r)-2 V_{T}(r)\right) \tilde{v}_{t(d)}^{3 p 1}(r)= \\
& \frac{2}{\sqrt{3}}\left[\left(u_{t(d)}(r)+\frac{1}{\sqrt{2}} w_{t(d)}(r)\right) \frac{\partial}{\partial r}\left(F_{\pi}^{1}(r)+\sqrt{2} F_{\rho}^{1}(r)-\sqrt{2} F_{\omega}^{1}(r)-\sqrt{2} F_{\rho}^{1^{\prime}}(r)\right)\right. \\
& +2 \sqrt{2}\left(F_{\rho}^{1}(r)-F_{\omega}^{1}(r)\right) \frac{\partial}{\partial r}\left(u_{t(d)}(r)+\frac{1}{\sqrt{2}} w_{t(d)}(r)\right) \\
& \left.-\frac{2 \sqrt{2}}{r}\left(F_{\rho}^{1}(r)-F_{\omega}^{1}(r)\right)\left(u_{t(d)}(r)-\sqrt{2} w_{t(d)}(r)\right)\right], \\
& \tilde{v}_{d}^{1 p 1^{\prime \prime}}(r)-\frac{2}{r^{2}} \tilde{v}_{d}^{1 p 1}+m_{N}\left(E-V_{C}(r)\right) \tilde{v}_{d}^{1 p 1}= \\
& \frac{2}{\sqrt{3}}\left[\left(u_{d}(r)-\sqrt{2} w_{d}(r)\right) \frac{\partial}{\partial r}\left(3 \chi_{\rho} F_{\rho}^{0}(r)-\chi_{\omega} F_{\omega}^{0}(r)\right)\right. \\
& -2\left(3 F_{\rho}^{0}(r)-F_{\omega}^{0}(r)\right) \frac{\partial}{\partial r}\left(u_{d}(r)-\sqrt{2} w_{d}(r)\right) \\
& \left.+\frac{2}{r}\left(3 F_{\rho}^{0}(r)-F_{\omega}^{0}(r)\right)\left(u_{d}(r)+2 \sqrt{2} w_{d}(r)\right)\right],
\end{aligned}
$$

where $F_{M}^{\Delta I}(r) \equiv g_{M N N} h_{M}^{\Delta I} f_{M}(r)$.

[1] C. S. Wood et al., Science 275 (1997) 1759.

[2] S. C. Bennett and C. E. Wieman, Phys. Rev. Lett. 82 (1999) 2484.

[3] A. R. Berdoz et al., Phys. Rev. Lett. 87 (2001) 272301.

[4] W. M. Snow et al., Nucl. Instrum. Methods 440 (2000) 729.

[5] B. Desplanques, J. F. Donoghue and B. R. Holstein, Ann. Phys. 124 (1980) 449.

[6] E. G. Adelberger and W. C. Haxton, Ann. Rev. Nucl. Part. Sci. 35 (1985) 501.

[7] B. Desplanques, Phys. Rep. 297 (1998) 1.

[8] J. Alberi et al., Can. J. Phys. 66 (1988) 542.

[9] B. Desplanques, Nucl. Phys. A 242 (1975) 425.

[10] M. Lacombe, B. Loiseau, J. M. Richard, R. Vinh Mau, J. Côté, P. Pirés and R. de Tourreil, Phys. Rev. C 21 (1980) 861.

[11] R. Machleidt, K. Holinde and Ch. Elster, Phys. Rept. 149 (1987) 1. 
[12] R. Machleidt, Adv. Nucl. Phys. 19 (1989) 189.

[13] R. B. Wiringa, V. G. J. Stoks and R. Schiavilla, Phys. Rev. C 51 (1995) 38.

[14] B. A. Craver, E. Fischbach, Y. E. Kim and A. Tubis, Phys. Rev. D 13 (1976) 1376.

[15] V. A. Knyazkov et al., Nucl. Phys. A 417 (1984) 209.

[16] B. H. H. McKellar and K. R. Lassey, Phys. Rev. C 17 (1978)842.

[17] F. Pauss, L. Mathelitsch, J. Côté, M. Lacombe, B. Loiseau and R. Vinh Mau, Nucl. Phys. A 365 (1981) 392.

[18] R. Schiavilla, J. Carlson and M. Paris, Phys. Rev. C 70 (2004) 044007.

[19] C. H. Hyun, T.-S. Park and D.-P. Min, Phys. Lett. B 516 (2001) 321.

[20] S. Morioka, P. Grange and Y. Avishai, Nucl. Phys. A 457 (1986) 518. 